\title{
Frozen Elephant Trunk and Antegrade Visceral Debranching in the Surgical Treatment of Type B Aortic Dissection: An Alternative Method
}

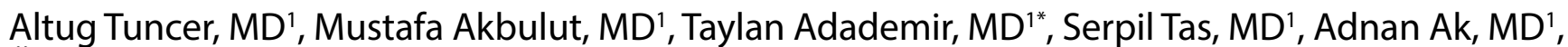 \\ Özgür Arslan, MD¹, Benay Erden, MD', Mesut Şişmanoğlu, MD² \\ ${ }^{1}$ Kartal Kosuyolu Heart and Research Hospital, Department of Cardiovascular Surgery, Istanbul, Turkey \\ 2 University of Duzce, Faculty of Medicine, Department of Cardiovascular Surgery, Duzce, Turkey
}

\begin{abstract}
Intervention is inevitable in complicated Type B aortic dissections. Classical surgical procedures and endovascular interventions are far from ideal treatments due to their high risk of periprocedural complications and mortality. There is often a need for alternative method in cases of difficult anatomy. We present the combined use of frozen elephant trunk and antegrade visceral debranching methods in the treatment of a 54-year-old male patient with complicated Type B aortic dissection.

Copyright (c) 2016 Science International Corp.
\end{abstract}

\section{Key Words}

Aortic dissection • Intervention • Surgery

\section{Introduction}

The classical surgery for Type B aortic dissection, which is performed by opening the thoracic and abdominal cavities and replacing the aorta and its branches, carries high morbidity and mortality and requires experience. Reports indicating mortality rates higher than $20 \%$, even from the most experienced centers, clearly demonstrate the need for alternative methods [1].

Although commonly used endovascular interven-

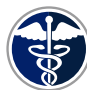

Fax +1 2037853552

E-Mail: aorta@scienceinternational.org

http://aorta.scienceinternational.org

\author{
(c) 2016 AORTA \\ Published by Science International Corp. \\ ISSN 2325-4637 \\ Accessible online at: \\ http://aorta.scienceinternational.org
}

tions have successful early- and intermediate-term results in cases of anatomical convenience, there are considerably high rates of retrograde dissection and endoleaks. While problems related to the intervention site, such as bilateral iliac artery stenosis or dissection, may prevent access to the lesion, solutions such as fenestrated and custom-made grafts may fail in cases with visceral organs supplied from a false lumen [2].

Our center implemented the novel procedure of combined use of the frozen elephant trunk (FET) procedure with antegrade visceral debranching as an alternative surgical technique for the treatment of complicated Type B aortic dissection. The indications, advantages, and limitations of this technique are presented in the context of the treatment of a case admitted to our clinic.

\section{Case Presentation}

A 54-year-old male patient having no systemic disease except hypertension was admitted to the emergency service of our hospital with a 1-week history of back and abdominal pain. Contrast-enhanced computed tomography (CT) angiography revealed a dissection flap starting $5 \mathrm{~mm}$ distal to subclavian artery,

* Corresponding Author:

Taylan Adademir, MD

Department of Cardiovascular Surgery

Kartal Kosuyolu Heart and Research Hospital

Cevizli, 34865 Kartal/Istanbul, Turkey

Tel. +90 216 5001500; Fax: +90 2164594321 ; E-Mail: taylanadademir@gmail.com 


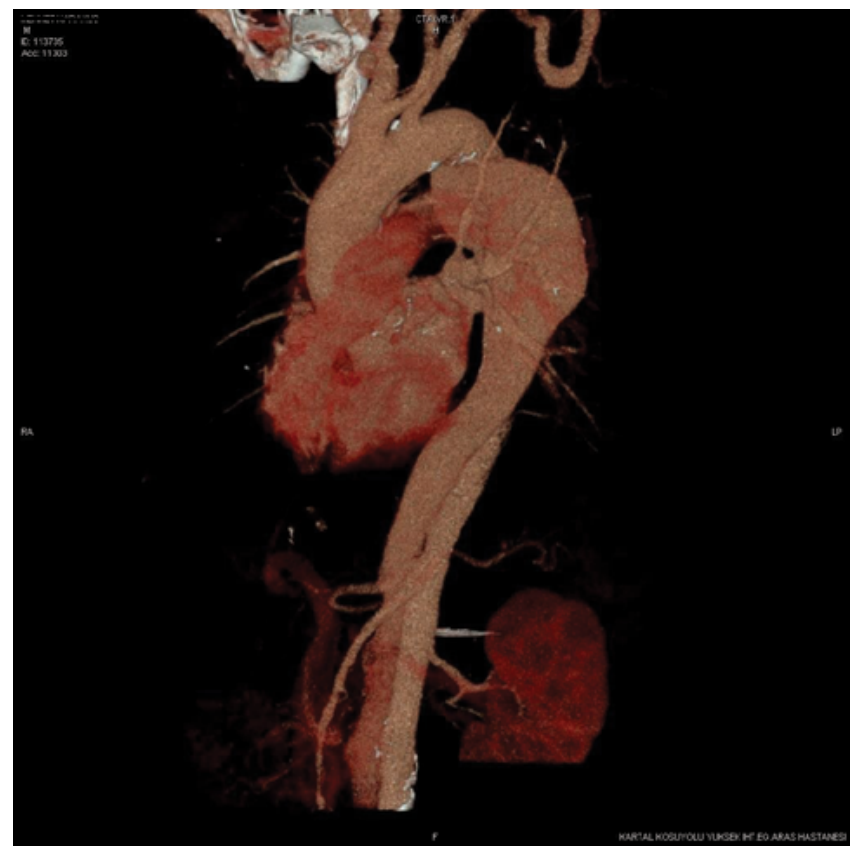

Figure 1. Preoperative three-dimensional computed tomography angiography image.

involving the celiac and right renal arteries and extending to both common iliac arteries (Figure 1). The diameter of the descending aorta was $68 \mathrm{~mm}$ at the level of the subclavian artery, $47 \mathrm{~mm}$ at the level of aortic truncus, and $32 \mathrm{~mm}$ at the level of diaphragm. The patient was referred to our department and hospitalized for advanced investigation and treatment. When his pain and signs of malperfusion worsened, he was taken to emergency surgery.

\section{Surgical Technique}

Surgery began after a central venous catheter was introduced through the right jugular vein, arterial pressure monitoring was started by placing a left radial line, cerebral pulse oximetry monitoring was accomplished, and a catheter was placed for cerebrospinal fluid pressure measurements. The right axillary artery was exposed. Following median sternotomy, the abdomen was opened by a midline incision, and the celiac trunk and right renal arteries were exposed and secured with surgical tape. Cardiopulmonary perfusion was started after systemic heparinization, right axillary arterial and two-stage unicaval venous cannulation. The patient was cooled. A Dacron Y-tube graft $(24 \times 12)$ was passed through the transverse si- nus. Perfusion pressure was reduced, and the proximal end of the Dacron tube graft was anastomosed end to side to the ascending aorta anterior to the transverse sinus under side clamping. When the trans-esophageal temperature reached $25^{\circ} \mathrm{C}$, an aortic cross clamp was placed, and the heart was arrested by antegrade tepid blood cardioplegia. After cardiac arrest, the cross clamp was moved to the brachiocephalic artery, and antegrade selective cerebral perfusion (flowrate: $10-15 \mathrm{~mL} / \mathrm{kg} / \mathrm{min}$ ) was established. Aortotomy was performed distal to the left subclavian artery, and it was observed that the dissection flap was 10-15 mm distal to the left subclavian artery. Single 4/0 pledgeted Prolene sutures were placed into the aorta distal to the subclavian artery. A $24 \times 150$ mm E-Vita Open Plus (JOTEC ${ }^{\circledR}$ GmbH, Germany) graft was introduced through the subclavian artery to the level of seventh vertebra and deployed according to the recommendations of the graft manufacturer. The proximal end of the graft was sutured to the aorta distal to the subclavian artery using preplaced Prolene sutures. After primary closure of the aortotomy, air was evacuated, the clamp was removed, and selective antegrade cerebral perfusion (58 minutes) was discontinued.

The implanted Dacron tube graft was passed through the aortic hiatus to the abdominal cavity. The proximal ends of celiac and right renal arteries were ligated, and distal ends were anastomosed end-toend with the legs of the Dacron graft. The surgery was completed in a total perfusion time of 140 minutes.

The patient's intensive care stay was prolonged due to respiratory complications, and he was transferred to the ward on postoperative day 6 . He was discharged uneventfully on postoperative day 15 .

In follow-up performed at 1, 6, and 16 months, the diameter of the descending aorta was measured as 58,56 , and $55 \mathrm{~mm}$, respectively, in control contrast-enhanced CT, and it was observed that descending aorta was completely thrombosed at the level of the graft and bypass grafts were patent. There were no clinical signs of malperfusion (Figure 2).

\section{Discussion}

Early intervention in cases with acute Type B dissection is not recommended because classical surgery 


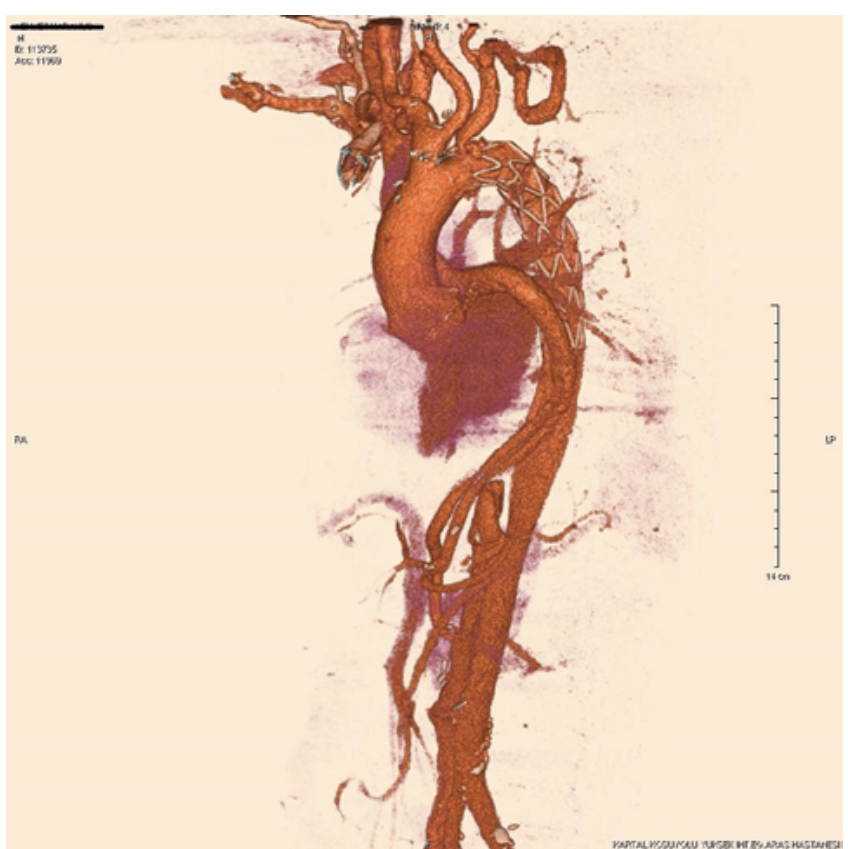

Figure 2. Postoperative three-dimensional computed tomography angiography image.

carries high morbidity, and endovascular interventions carry risk of retrograde dissection and have unknown long-term results. Surgical or endovascular interventions are indicated in complicated cases (i.e., in cases with rupture, treatment-resistant hypertension, uncontrolled pain and malperfusion syndrome) $[3,4]$. There is an increasing trend for endovascular interventions in recent years as they promise more successful results than medical and classical surgical treatment in cases with life-threatening ruptures [5]. Besides problems related to the intervention site like bilateral iliac artery stenosis or dissection, which may make the intervention impossible, the routine use of this intervention is restricted due to the risk of retrograde thoracic aortic dissection during and after the procedure and Type la endoleaks [6].

Another vulnerable point in endovascular interventions is the presence of a dissection flap involving visceral artery branches in patients with malperfusion. Perfusion defects are among the most important factors increasing mortality in these patients [1, 7]. In the presence of dynamic obstruction, organ perfusion and symptoms can be improved after an endovascular procedure, but the treatment options for patients with continued malperfusion or static ob- struction are segmentation of the dissection flap, interventions using noncovered stents, or extra-anatomic bypass $[8,9]$. Although the early results of noncovered stents are encouraging, long-term outcomes and problems related to their use are not yet known.

We thought that restoration of blood flow of malperfused organs via the dissected iliac artery was not convenient in the present case, and access by the femoral route was not safe due to bilateral iliac artery dissection. Extra-anatomic bypass solutions performed to the malperfused organ supplied from a dissected artery may be problematic because of structural problems and turbulence due to the retrograde flow. Although high patency rates are reported in the literature, potential problems related to retrograde flow are not known [10].

To the best of our knowledge, this case is the first isolated Type III aortic dissection case in the literature in which antegrade perfusion of the malperfused organs was provided from the ascending aorta (preserved from dissection) and the intimal rupture was closed with an open FET procedure instead of using the unsafe femoral route.

Our surgical method carries the potential to answer many problems. It provides direct visualization of the primary tear in the aorta. It permits closure of the rupture with a graft, completely eliminating Type la endoleaks and the risk of retrograde dissection by suturing the proximal end of the graft to the aorta. It provides convenience for a probable second surgery by the graft placed to the descending aorta. It achieves simultaneous perfusion of all visceral organs by antegrade (according to the direction of the flow) debranching. Thus, we believe there are multiple advantages of this method. The need for cardiopulmonary bypass and selective antegrade cerebral perfusion for the application of the procedure are the greatest disadvantages.

In conclusion, the FET method is an alternative in the treatment of cases with complicated Type III aortic dissection in which the femoral route cannot be used. This method, while preventing the proximal spread of dissection, removes the risk of Type 1a endoleaks. By stabilizing the dissection flap in the descending aorta, the graft preserves perfusion of the organs supplied from the true lumen. After the procedure, the 
visceral organs with malperfusion can be supplied in the normal flow direction using the vessel preserved from dissection (antegrade) in the same session. The greatest limitation is the need for cerebral perfusion and cardiopulmonary bypass during the procedure. We suggest that this method can be an alternative in selected cases.

\section{Conflict of Interest}

The authors have no conflict of interest relevant to this publication.

\section{Comment on this Article or Ask a Question}

\section{References}

1. Bozinovski J, Coselli JS. Outcomes and survival in surgical treatment of descending thoracic aorta with acute dissection. Ann Thorac Surg. 2008;85:965-970. DOI: 10.1016/j.athoracsur.2007.11.013

2. Kitagawa A, Greenberg RK, Eagleton MJ, Mastracci TM, Roselli EE. Fenestrated and branched endovascular aortic repair for chronic type B aortic dissection with thoracoabdominal aneurysms. J Vasc Surg. 2013;58:625-634. DOI: 10.1016/j. jvs.2013.01.049

3. Akin I, Kische $\mathrm{S}$, Ince $\mathrm{H}$, Nienaber $\mathrm{CA}$. Indication, timing and results of endovascular treatment of type B dissection. Eur J Vasc Endovasc Surg. 2009;37:289-296. DOI: 10.1016/j.ejvs.2008.12.004

4. Atkins MD, Black JH, Cambria RP. Aortic dissection: perspectives in the era of stent-graft repair. J Vasc Surg. 2006;43 Suppl A:30A-43A. DOI: 10.1016/j. jvs.2005.10.052

5. Kitagawa A, Greenberg RK, Eagleton MJ, Mastracci TM, Roselli EE. Acute complicated and uncomplicated type III aortic dissection: an endovascular perspective. Semin Thorac Cardiovasc Surg. 2009;21:373-386. DOI: 10.1053/j.semtcvs.2009.11.008

6. Chen SL, Zhu JC, Li XB, Ye F, Zhang JJ, Liu $\mathrm{ZZ}$, et al. Comparison of long-term clinical outcome between patients with chronic versus acute type $B$ aortic dissection treated by implantation of a stent graft: a single-center report. Patient Prefer Adherence. 2013;7:319-327. DOI: 10.2147/PPA. S39012

7. Hagan PG, Nienaber CA, Isselbacher EM, Bruckman D, Karavite DJ, Russman PL, et al. The International Registry of Acute Aortic Dissection (IRAD): New insights into an old disease. JAMA. 2000;283:897-903. DOI: 10.1001/jama.283.7.897

8. Vedantham $S$, Picus $D$, Sanchez $A L$, Braverman A, Moon MR, Sundt T, et al. Percutaneous management of ischemic complications in patients with type- $B$ aortic dissection. J Vasc Interv Radiol. 2003;14:181-193. PMID: 12582186
9. Uchida N, Shibamura H, Katayama A, Aishin K, Sutoh M, Kuraoka M. Surgical strategies for organ malperfusions in acute type B aortic dissection. Interact Cardiovasc Thorac Surg. 2009;8:75-78. DOI: 10.1510/ icvts.2008.186247

10. Chiesa R, Tshomba Y, Melissano G, Logaldo $D$. Is hybrid procedure the best treatment option for thoraco-abdominal aortic aneurysm? Eur J Vasc Endovasc Surg. 2009;38:26-34. DOI: 10.1016/j.ejvs. 2009.03.018

Cite this article as: Tuncer $A$, Akbulut $M$, Adademir T, Tas S, Ak A, Arslan Ö, Erden B, Şişmanoğlu M. Frozen Elephant Trunk and Antegrade Visceral Debranching in the Surgical Treatment of Type B Aortic Dissection: An Alternative Method. AORTA (Stamford). 2016;4(5):167-171. DOI: $\quad$ http://dx.doi.org/10.12945/j. aorta.2016.15.040

\section{EDITOR'S QUESTIONS:}

\section{Did this patient manifest clinical malperfusion or just radiographic?}

The patient was complaining of abdominal pain, presumably due to ischemia triggered by dynamic and static blood flow obstruction.

2. Tell us why you use the transverse sinus, rather than an anterior trajectory from the ascending aorta.

The Dacron graft was passed through the transverse sinus to avoid kinking during its course in the mediastinum.
3. Your technique may have benefits even if there is good femoral access (e.g., fixing the FET fully, preventing retrograde dissection). Should your technique be preferred even if there is good femoral access?

In complicated type $B$ acute aortic dissections, there is a risk of retrograde ascending dissection during or after the TEVAR procedure if the ascending aorta or aortic arch diameters are larger than 4 centimeters [1]. Therefore, we propose that our technique should be preferred in patients with a retrograde dissection risk even if there is good femoral access.

1. Williams $J B$, Andersen ND, Bhattacharya $S D$, 
Scheer E, Piccini JP, McCann RL, et al. Retrograde ascending aortic dissection as an early complication of thoracic endovascular aortic repair. J Vasc Surg. 2012;55:1255-1262.

4. Describe the aortic anatomy we see around the diaphragm in the post-operative 3D reconstruction?

Both true and thrombosed false lumens were seen; however, expansion of true lumen was not yet sufficient. Considering that there are no re-entries down to the level of the celiac trunk, we can say that the procedure was successful.
5. For the "tacking" sutures that you place beyond the subclavian and use to perfuse the graft, can you place these around the full circumference of the descending aorta? How?

Those Prolene sutures were placed around the full circumference of the descending aorta. However, the first four were placed to secure the posterior wall prior to introduction of the frozen elephant trunk graft because the posterior wall had limited accessibility due to incomplete transection of the descending aorta. The remaining sutures were placed later on, and the FET graft was fixed. 